\title{
A
}

\section{Introduction to the Quantum Realm}

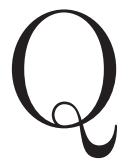

UANTUM MECHANICS describes nature at very small scales - at the atomic and subatomic levels, but quantum effects have been observed in large molecules as well. The idea that our everyday world is made from small particles, which we call atoms, dates to the ancient Greeks. Today we think of atoms as small spherical objects that are ten-billionths of a meter in diameter. This measurement $\left(10 \times 10^{-10} \mathrm{~m}\right)$ is so common that it has a special name - the Angstrom - and a special symbol, $\AA$. Objects that are angstrom-sized behave very differently than objects the size of everyday objects like tennis balls and automobiles. Because humans grow up looking at and manipulating everyday objects, most people do not have any intuition about how angstrom-sized objects behave until they have been educated in modern physics. While Democritus of Abdera came up with the idea of atoms more than 2500 years ago and our understanding of chemistry has evolved over many centuries, our understanding of quantum mechanics was developed mostly over the past 125 years.

Information theory concerns how information is stored, communicated and quantified. Although humans have been storing and communicating information for thousands of years, our mathematical understanding of what information actually is dates to a paper by Claude Shannon from 1948, "A Mathematical Theory of Communication." Among other things, the paper introduced the term "bit,"

${ }^{1}$ Shannon, "A Mathematical Theory of Communication" (1948). 
short for binary digit, as the fundamental unit of information. Much of what is known about the nature of information - including codes, compression and encryption - was first worked out in the 1940s.

As its name implies, QIS combines these two disciplines. Broadly, QIS is the study of approaches that combine knowledge of how quantum effects can be used to measure, sense, communicate, and compute.

This appendix is the first of two that are intended to provide an introduction to quantum mechanics for policymakers who may be generally knowledgeable about our technological world, but who (realistically) did not progress beyond algebra and introductory physics in high school or college. This chapter explains quantum scale and starts an exploration as to why effects at the quantum scale are so radically different from humans' day-to-day experience. Appendix B explores more of quantum mechanics and shows how that theory applies to information science. Readers who want to jump directly to a functional understanding of what quantum technologies enable should review Part 1, "Quantum Technologies."

\section{A.1 The Quantum World: A Brief Introduction}

Albert Einstein, Niels Bohr, Max Planck, Werner Heisenberg, and others led what is known as the "first quantum revolution" when they created quantum mechanical theory in the early twentieth-century. Their work was sparked not by a desire to understand things that were very small, but to explain phenomena that could be measured in the world of the 1890s. In short order, they realized that explaining these phenomena required rethinking their understanding of matter, energy, and even time. To do so, they used a combination of physical experiments that were carefully constructed so that their results depended upon the interaction of quantum forces.

The experiments and their interpretations made by these physicists had profound consequences. Fission and fusion bombs are quantum weapons. Other quantum devices powered by the first quantum revolution include the atomic clock, lasers, the transistor, and medical imaging technology, such as magnetic resonance imaging (MRI).

The pace of QIS innovation is increasing, so much so that Jonathan Dowling and Gerald Milburn have labeled the current age the "second quantum revolution." In this second revolution, technologies leverage the special physics of the very small to measure physical 
phenomena and time more precisely (quantum metrology), to create imagery or otherwise sense phenomena invisible to ordinary sight (quantum sensing), to communicate information, including more secure encryption keys (quantum communications), and to engage in computing (quantum computing). ${ }^{2}$

Writing in 2003, Dowling and Milburn attributed the second revolution to the need for miniaturization and to the potential performance enhancements that QIS provided over technologies governed by classical physics. Today, miniaturization and performance continue to be important driving factors, but other political imperatives and technology developments have emerged to contribute to the second revolution.

Quantum theory seems perplexing because humans have no experience of the subatomic world in daily life. Quantum physics is counterintuitive and difficult to grasp; unfortunately, this means that the label quantum frequently becomes a smokescreen for claptrap. When learning about QIS, it is important to distinguish reasoned discussion of quantum effects from quantum fiction designed to entertain, confuse, or distract.

Quantum fiction is readily seen in Hollywood movies where a superhero might pass her hand through a wall, explaining "well, we are mostly made up of empty space" and then perhaps adding a throwaway explanation that her hand is making use of "quantum tunneling." This seems reasonable, because the atomic nucleus is in fact tiny compared to the size of atom and quantum tunneling is a real phenomenon. Quantum tunneling appears to allow particles to skip through barriers, it is the basis of scanning tunneling microscopy, and it presents a fundamental limit for how small the features of integrated circuit transistors might actually get. But quantum tunneling only happens at the subatomic scale. In real life, a superhero cannot phase through a wall because the electrons in the hero's hand repel the electrons in the wall. That is why the quantum fiction in superhero movies relies on computer graphics to add the visual effects, rather than relying on quantum physics.

\section{A.2 Terminology, Size, and Frequency}

This section introduces the terminology of modern physics and conveys a sense of the sizes involved.

${ }^{2}$ Dowling and Milburn, "Quantum Technology: The Second Quantum Revolution" (2003). 


\section{A.2.1 The Atom}

Hydrogen is the simplest atom, with a single negatively charged electron orbiting around a positively charged nucleus that contains a single proton. Because it has a single proton, the hydrogen atom is said to have an atomic weight of 1 . Hydrogen gas is a molecule that consists of two hydrogen atoms: the two nuclei each consists of a single proton, and the two electrons are shared between them, forming what chemists call a covalent bond. (Although the chemical formula for elemental hydrogen is $\mathrm{H}_{2}$, it is sometimes written $\mathrm{H}: \mathrm{H}$ to emphasize that the two electrons are shared.)

A small fraction of the hydrogen on the planet has both a positively charged proton and a neutrally charged neutron in its nucleus: this kind of hydrogen is called deuterium and it has an atomic weight of 2. Water made from deuterium is called heavy water and played a role in the German atomic bomb program in World War II because it can be used as a moderator to sustain a nuclear chain reaction, a critical step in producing plutonium. It is also used in medical research, to measure food intake and energy balance.

A third kind of hydrogen, called tritium, has two neutrons; tritium is highly radioactive because the atom's nucleus has twice as many neutrons as protons. (Nuclei become unstable if the neutron/proton ratio is more than $1.5: 1$.) Tritium has been used in self-illuminating mechanical watch dials and as a tracer in medical diagnosis. It is also an ingredient in certain kinds of nuclear weapons.

Quantum mechanics describes electrons, protons and neutrons with mathematical equations that define a probability distribution. Instead of thinking of electrons whizzing around the nucleus like planets around the Sun, think instead of an electron cloud surrounding the nucleus, like a swarm of bees buzzing around a hive. But even that analogy is flawed. Mathematically, the hydrogen's electron is better described with equations that describe a wave centered on the atomic nucleus, like the vibrating surface of a bell that has been struck. The equation describes how the electron's energy changes when it absorbs light.

Quantum mechanics also describes how hydrogen atoms resemble a spherical shell with a diameter of approximately $1.1 \AA$ centered on the hydrogen nucleus. The nucleus is described with a similar equation, except that it has a diameter of $0.000017 \AA\left(1.7 \times 10^{-5} \AA\right.$, or $\left.1.7 \times 10^{-15} \mathrm{~m}\right)$. 
Hydrogen's electrons can be anywhere, no space within the atom is strictly empty. Look at an even smaller scale, and even the "empty space" within the atom - as well as the empty space between atoms - may be filled with observable space-time fluctuations - quantum foam - in which mass and energy is created and destroyed in a manner that is consistent with the Heisenberg Uncertainty Principle.

\section{A.2.2 Quantum Sizes}

The previous section uses measurements like $1.1 \AA$ and $10 \times 10^{-15} \mathrm{~m}$ without much reflection; this section attempts to provide a better understanding of quantum sizes.

In the classic 1977 short movie Powers of Ten and the Relative Size of Things in the Universe and the Effect of Adding Another Zero, the noted twentieth-century designers Charles and Ray Eames take the viewer on a voyage through 46 orders of magnitude. ${ }^{3}$

When the movie starts, the field of view is 1 square meter (1.09 yards) and shows a man and woman at rest on a blanket on the western shore of Lake Michigan. The field of view then zooms out, a factor of 10 every 10 seconds. At 20 seconds, the field of view is 100 meters, showing the entire field, at 30 seconds the $1000 \mathrm{~m}$ field of view shows several blocks, and so on.

Scientists and engineers commonly use exponents to describe large numbers. The measurement $1000 \mathrm{~m}$ can be written as $10^{3} \mathrm{~m}$ or as $1 \mathrm{~km}$ (1 kilometer). The notation $10^{3}$ literally means "the number ten multiplied by itself three times," or $10 \times 10 \times 10$. Scientific notation is useful for measurements like $10^{10} \mathrm{~m}$ (the distance the Earth travels through space in about four days), $10^{20} \mathrm{~m}$ (the scale of the structure of the Milky Way Galaxy and its rich brotherhood of stars) and $10^{24} \mathrm{~m}$, the maximum scale shown in the Eames movie. Today we believe that the size of the observable universe is 93 billion light years, or $3.6 \times 10^{25}$ meters. Many computer programs use the letter "E" to represent scientific notation, so the reader may encounter the measurement written as "3.6E25m."

The second half of the Eames film returns to the couple in Burnham Park and then zooms off in the other direction, everything in the frame getting smaller by a factor of 10 every 10 seconds. Twenty seconds into the second half, the field of view is $10^{-2} \mathrm{~m}$ across (also called 1 centimeter, or one hundredth of a meter). The frame shows

\footnotetext{
${ }^{3}$ The nine-minute Eames film is online www.eamesoffice.com/the-work/powers-of -ten/.
} 


\section{-Understanding Negative Exponents-}

Mathematicians define the number $10^{0}$ as 1 , which might seem strange. How can you multiply anything by itself zero times? Should not the answer be zero, not one? This is an example of a model that works well in one domain, but fails when applied to another.

Because the addition of exponents can be defined in terms of multiplication, subtracting exponents is defined as division. Just as 1000 divided by 100 is $10,10^{3}$ divided by $10^{2}$ is $10^{1}$. That is, $10^{3} \div 10^{1}=10^{3-2}=10^{1}=10$. It then follows that $10^{0}$ is 1 . (This also works if you think of the exponent $x$ in the equation $10^{x}$ as the number of 1 followed by $x$ zeros, which shows the advantage of having a superior mental model.)

Negative exponents extend this idea in the other direction. The number $10^{-1}$ is the same as the number 0.1 or $\frac{1}{10}$. More generally, $10^{x}=\frac{1}{10^{x}}$.

a patch of skin on the man's hand. At 50 seconds the scale is $10^{-5} \mathrm{~m}$, or 10 micrometers (also called microns), which is the size of a white blood cell. At $10^{-10} \mathrm{~m}(1 \AA)$ the screen fills with the electron shell of a hydrogen atom. At $10^{-15} \mathrm{~m}$, which the film calls a "fermi" (a unit of measure named for Enrico Fermi but not widely used), the screen shows a proton and a neutron, two of the building blocks of matter. The film stops at $10^{-16} \mathrm{~m}$, which the narrator explains is the scale of quarks, electrons, and positrons.

As the preceding paragraphs demonstrate, one of the reasons policy specialists find it difficult to digest quantum literature is that the same measurements can be described many different ways. For example, the nitrogen atoms used for quantum sensing discussed in this book have a diameter of $1.12 \AA$, but that measurement might alternatively be reported as a radius of 56 picometers $(\mathrm{pm}), 0.056$ nanometers $(\mathrm{nm})$ or $5.6 \times 10^{-11} \mathrm{~m}$.

In addition, scientists typically describe the measurement of a sphere with its radius (the distance from the center to the surface), rather than the diameter, because the equations that describe the properties of circles and spheres are simpler when based on $r$ (the radius) rather than $d$ (the diameter). But for people who think of atoms as tiny tennis balls, the concept of radius can be confusing, 
because we tend to think of tennis balls as spheres which have an official diameter of $6.54-6.86 \mathrm{~cm}$ (as defined by the International Tennis Federation) - and not as spheres that have an official radius of $3.27 \times 10^{-2} \mathrm{~m}$ to $3.43 \times 10^{-2} \mathrm{~m}$. (Of course, both measurements are exactly the same.)

At quantum scales, nature is probabilistic and objects have behaviors reminiscent of both waves and particles. This differs from how objects behave at the scale of real tennis balls, rackets and courts. The way these objects behave guides our intuition and, as a result, shaped the development of what is called classical physics. In our ordinary lives, one can determine how objects will act by knowing their mass, inertia, and so on. At a quantum scale, reality is governed by probability. That is, one can make predictions about the location of subatomic particles but these predictions are probabilities. As such, quantum science is as unsettling as is it profound.

\section{A.2.3 Light}

The fundamentals of light are a focus of early education. For example, many students in high school will learn that sunlight is actually a mixture of all the colors in the rainbow, and what we call a "rainbow" is actually drops of rain acting like a prism, splitting sunlight into its component colors. On the other hand, students who take theater class will learn that what looks like white light can be produced by mixing red, green, and blue light together. (That works because most people's eyes have three kinds of color-sensing cells, sensitive to red, green, and blue light respectively.) Light can be filtered by color: shine white light into a red filter, and red light comes out the other side. But light of one color cannot be changed into another color: pass red light into a blue filter, and nothing comes out.

For many years scientists were confused about the fundamental nature of light: some scientists, like Isaac Newton, thought light was made out of tiny objects he called corpuscles. Other scientists like Thomas Young thought that light was actually some kind of wave traveling through some kind of medium variously called the ether, also written aether, ather (or even $\alpha i \theta \eta \rho$ if you happen to write in ancient Greek).

If you use a prism to produce a rainbow, you will discover that some of the Sun's energy extends on both ends beyond the familiar red-orange-yellow-green-blue-indigo-violet (a.k.a. ROYGBIV) color chart. Place your hand to the left of the red and your hand will grow 


\section{-Through a Glass Darkly...}

QIS forces us to understand just how limited human perception is. As humans, we indeed see the world through a glass darkly. Consider what we see of the world - the visible light spectrum (see below). The quantum realm exists mostly outside the world of human experience. QIS and resulting quantum technologies are counterintuitive because there are few, fleeting moments when humans see quantum effects. Our entire experience is based on the physics of relatively large objects.

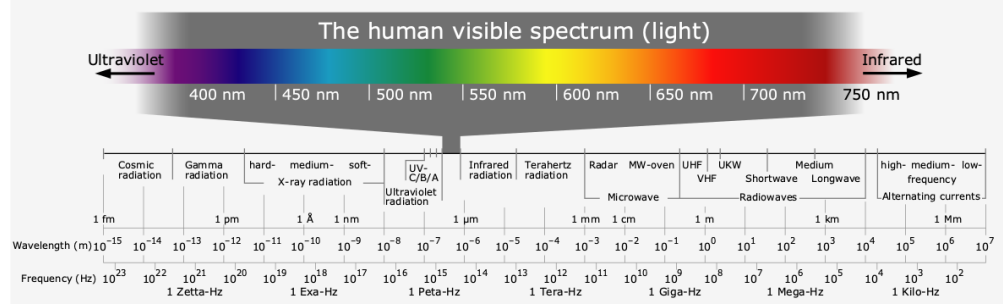

Will better understanding the physics of the small change how we perceive our own greatness, and even relevance? The same passage of the Bible that gives us the lovely metaphor of seeing through a glass darkly, a reference to the imperfect mirrors of antiquity, explains the concept that our perception of god is incomplete: For we know in part, and we prophesy in part. But when that which is perfect is come, then that which is in part shall be done away.

Quantum physics is replacing the imperfect mirror of classical mechanics. That imperfect mirror allowed us to leave many questions to prophesy. As the quantum physics mirror is perfected, what will be left to prophesy?

Sidebar 1 . Visible light is a tiny part of the electromagnetic spectrum. Image CC BY-SA 4.0 by Wikimedia user Horst Frank.

warm from infrared light, which is too far red for the eye to see. Sir William Herschel discovered this effect in 1800, although he used a thermometer and not his hand.

Place your hand to the right of the violet and you will get a suntan, and then a burn, from the ultraviolet light. Johann Wilhelm Ritter discovered ultraviolet this way in 1801, although Ritter used 
silver chloride, a chemical that turns black when it is exposed to sunlight (and was the basis of the wet chemistry used in photography for nearly two centuries). Ritter discovered that silver chloride turned black faster with blue light than with red light - and faster still when placed past the violet edge of a spectrum created with sunlight and a glass prism.

What is called visible light is actually just a tiny part of the electromagnetic spectrum, which includes radio waves, microwaves, infrared rays, visible light, ultraviolet light, X-rays, and gamma rays (see Table A.2). As humans, we perceive the reality of the physical world through a glass darkly.

\section{A.2.4 Quantum Speeds}

Light is one of the best understood quantum objects, in part because of its simplicity, in part because it is the easiest to study, and in part because humans can observe it. In ancient times people thought that light moved instantly, but in the 1670s the Danish astronomer Ole Rømer determined light must have a finite speed based on his observations of eclipses of Jupiter's moon Io. Rømer's estimation of the speed of light was about 220000 kilometers per second, roughly three-quarters the actual value of $299792 \mathrm{~km} / \mathrm{s}$ in vacuum.

All colors of light travel at the same speed, as do radio waves, microwaves, and gamma rays. It turns out that a beam of monochromatic light also has a wavelength and a frequency. They are related by this equation:

$$
c=\lambda f
$$

where:

$$
\begin{aligned}
c= & \text { the speed of light (roughly } 300000 \mathrm{~km} / \mathrm{s} \text { ) } \\
\lambda= & \text { the wavelength of light } \\
f= & \text { the frequency of the light, measured in cycles } \\
& \text { per second }(\mathrm{Hz}) .
\end{aligned}
$$

That is, the wavelength times the frequency is equal to the speed of light. Since the speed of light is constant (it is a sort of universal speed limit), light with small wavelength has a high frequency, and light with large wavelength has a small frequency. 
Table A.1. The electromagnetic spectrum.

\begin{tabular}{lll} 
Kind of light & Wavelength & Frequency \\
\hline Longwave radio & $3 \times 10^{8} \mathrm{~m}$ & $10^{0} \mathrm{~Hz}=1 \mathrm{~Hz}$ \\
AM radio waves & $3 \times 10^{2} \mathrm{~m}$ & $10^{6} \mathrm{~Hz}=100 \mathrm{Kilohertz}$ \\
FM radio waves & $3 \times 10^{0} \mathrm{~m}=3 \mathrm{~m}$ & $10^{8} \mathrm{~Hz}=100$ Megahertz \\
Microwaves & $3 \times 10^{-2} \mathrm{~m}=3 \mathrm{~cm}$ & $10^{10} \mathrm{~Hz}=10$ Gigahertz \\
Near Infrared & $3 \times 10^{-6} \mathrm{~m}=3 \mu \mathrm{m}$ & $10^{14} \mathrm{~Hz}=10$ Terahertz \\
Visible & $380-740 \mathrm{~nm}$ & $405-790$ Terahertz \\
Ultraviolet & $3 \times 10^{-8} \mathrm{~m}=30 \mathrm{~nm}$ & $10^{16} \mathrm{~Hz}=1$ Petahertz \\
X-rays & $3 \times 10^{-10} \mathrm{~m}=3 \AA$ & $10^{18} \mathrm{~Hz}=100$ Petahertz \\
Gamma rays & $3 \times 10^{-14} \mathrm{~m}$ & $10^{22} \mathrm{~Hz}$
\end{tabular}

Table A.2. The visible electromagnetic spectrum.

\begin{tabular}{lll} 
Color & Wavelength & Frequency \\
\hline Violet & $380-450 \mathrm{~nm}$ & $680-790 \mathrm{THz}$ \\
Blue & $450-485 \mathrm{~nm}$ & $620-680 \mathrm{THz}$ \\
Cyan & $485-500 \mathrm{~nm}$ & $600-620 \mathrm{THz}$ \\
Green & $500-565 \mathrm{~nm}$ & $530-600 \mathrm{THz}$ \\
Yellow & $565-590 \mathrm{~nm}$ & $510-530 \mathrm{THz}$ \\
Orange & $590-625 \mathrm{~nm}$ & $480-510 \mathrm{THz}$ \\
Red & $625-740 \mathrm{~nm}$ & $405-480 \mathrm{THz}$
\end{tabular}

Table A.1 shows the wavelengths and frequencies for various kinds of light. Notice that invisible light does not follow the convention of falling within even powers of 10 . Unfortunately visible light does not fit neatly into this table; our eyes evolved to perceive light in the relatively tiny range of light that has wavelengths of $380 \mathrm{~nm}$ to $740 \mathrm{~nm}$, as shown in Table A.1. ${ }^{4}$

This exposition reveals an important point: the quantum realm and almost all of its effects happen outside human perception. We perceive the world through a glass darkly - a glass that only reveals

\footnotetext{
${ }^{4}$ Not every human's eyes work the same way. There is a version of so-called "color blindness" in which the blue cones are sensitive in the ultraviolet, and there are some humans who have four color receptors, a condition called tetrachromacy. Such people, who are exceedingly rare, see a richer pallet of colors.
} 
visible light. The quantum world and quantum effects typically require special equipment to perceive and to manipulate. As a result, we have little day-to-day experience with the quantum world, and its attributes are thus counterintuitive and take work and study to learn.

This appendix introduced the basics of the quantum realm. The appendix covered the reasons why quantum information science is exciting, the relative sizes of quantum phenomena, fundamental properties of light, and the idea that everything has wave- and particle-like properties. This foundation is necessary for the next appendix, which turns to quantum effects: wave mechanics, the uncertainty principle, polarization, entanglement, superposition, and the "cat state." 
